\title{
ANALYSIS OF THE STATIC AND DYNAMIC MECHANICAL BEHAVIOR OF A TIBIAL BONE-KNEE IMPLANT ASSEMBLY WITHOUT A TIBIAL EXTENSION
}

\author{
Mihai-Constantin Balaşa ${ }^{1[2]}$ and Viviana Filip ${ }^{2}$ \\ ${ }^{1}$ Macartney Hydraulics A/S, DK-7620 Lemvig, Denmark \\ ${ }^{2}$ Valahia University of Targoviste, Doctoral School of Engineering Sciences, 130105, Targoviste, Romania \\ E-mail: vfilip@yahoo.com
}

\begin{abstract}
The ever-growing number of total knee replacement (TKR) surgical procedures has brought a proportional increase in the number of knee replacement revision operations. Among the causes of the early failure of a knee implant are the weakening and displacement of the tibial component. To avoid revision surgery and extend the lifespan of the implant, it is really useful to perform a finite element analysis in order to identify the areas that may be prone to cause the weakening of the tibial component.

The purpose of this paper is the virtual modeling of the tibia-knee implant assembly and the analysis of its mechanical behavior both in the static and the dynamic regime. For this purpose, we will look at the case of an implant without tibial extension and analyze the healthy bone in comparison with the osteoporotic bone. The results from the static stress, fatigue, linear and nonlinear dynamic stress tests confirm that the healthy bone has a higher mechanical strength and a lower degree of damage than the affected bone, under the same stress conditions.
\end{abstract}

Keywords: CAD Techniques, Tibial Bone, Knee Implant, Finite Element Analysis.

\section{Introduction}

Currently, the choice of the implant type for a knee replacement (Fig. 1) is made after medical imaging procedures.

A radiography machine provides $2 \mathrm{D}$ images (with the final result consisting in a negative photograph) that make it possible to establish the diagnosis and choose the most appropriate implant that will be used to replace the knee joint.

The factors the implant choice depend on and the surgeon will have to consider before scheduling the procedure are related to the patient's age, weight, activity, and health condition. Just as the wear occurring in the natural joint leads to the necessity to replace the joint, so the wear of the implant may require possibly a second procedure, called revision surgery.

To extend the lifespan of the primary implant by choosing the best option available and in order to avoid or postpone as much as possible the revision surgery [1], it would be really useful for the surgeon to know, before the primary surgery [2,3], how the implanted bone behaves in time.

This type of analysis is possible with the help of CAD techniques and is the subject of this paper.

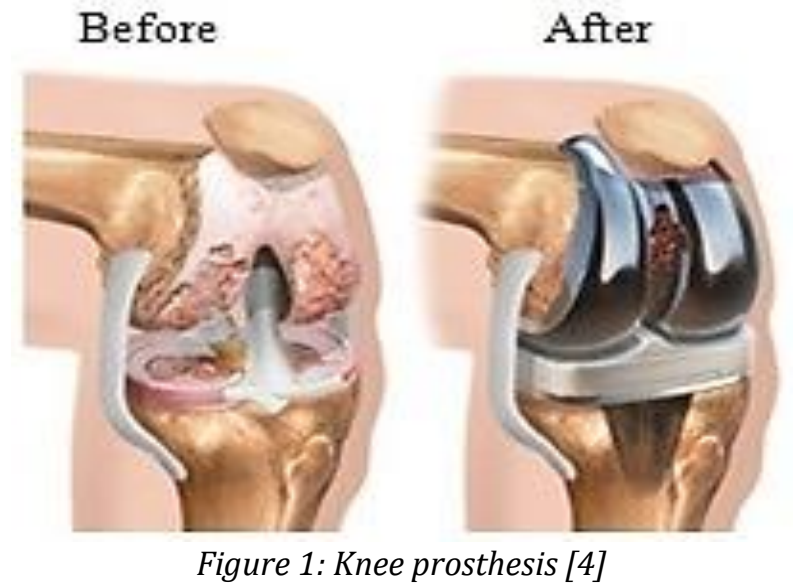

In order to study the static and dynamic mechanical behaviour of the tibia-knee implant assembly, we used the Solid Works software to create the virtual model of a $320 \mathrm{~mm}$-long tibial bone (based on an existing physical model which was previously 3D scanned) with the thickness of the cortical bone (i.e., the compact outer surface) varying between $0.8 \mathrm{~mm}$ (at the extremities, in the knee and the ankle areas) and $4 \mathrm{~mm}$ (in the middle area) [5]. The same software was used to create the components of the implant (based on an existing physical model of a widely-used standard prosthesis), including the cement used for fixation 
[6]. Then we used the finite element method to study the mechanical behavior of the bone-implant assembly.

\section{Working Method and Resources Used}

After creating the virtual model of the tibia and knee implant, we produced the tibial bone-implant assembly (Fig. 2-a), seeking the correct positioning of the implant on the tibial plateau. The tibial component of the implant is aligned correctly by dividing hypothetically the tuberosity into three parts (Fig. 2-b): the medial part (the inside of the bone, i.e. the side facing the other leg), the central part (the middle) and the lateral part (the outward side). A line is drawn that separates the medial part from the central part, then the tibial metal component of the implant (i.e. the tibial plateau) is positioned on the borderline.

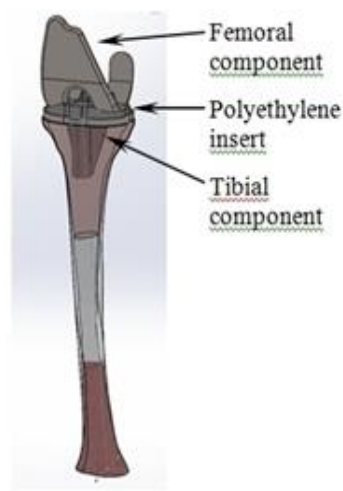

a)

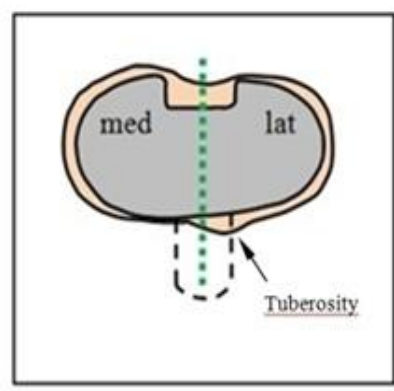

b)
Figure 2: Knee Implant, a) Implant components, b) Tibial Alignment

Next, we assigned material properties to each of the assembly's components: cortical bone, spongy bone, polyethylene insert, tibial metal component, and fixation cement - as follows:

For the healthy cortical bone: Young's modulus E $=1700 \mathrm{~N} / \mathrm{mm}^{2}$, Poisson's ratio $=0.2$, density $=800$ $\mathrm{kg} / \mathrm{m}^{3}$, Tensile strength $1.2 \mathrm{~N} / \mathrm{mm}^{2}$, Compressive strength $1.9 \mathrm{~N} / \mathrm{mm}^{2}$.

For the healthy spongy bone: $\mathrm{E}=700 \mathrm{~N} / \mathrm{mm}^{2}$, Poisson's ratio $=0.2$, density $=600 \mathrm{~kg} / \mathrm{m}^{3}$, Tensile strength $1.3 \mathrm{~N} / \mathrm{mm}^{2}$, Compressive strength $1.8 \mathrm{~N} /$ $\mathrm{mm}^{2}$.

For the polyethylene insert we selected the HighDensity Polyethylene option from the list of materials and assigned the following default parameters: Young's modulus $\mathrm{E}=1070 \mathrm{~N} / \mathrm{mm}^{2}$, Poisson's ratio $=0.41$, density $=952 \mathrm{~kg} / \mathrm{m}^{3}$

For the tibial component we selected the Ti-6Al$4 \mathrm{~V}$ alloy from the list of materials and assigned the following default parameter: Young's modulus E = $104800 \mathrm{~N} / \mathrm{mm}^{2}$, Poisson's ratio $=0.31$, density $=$ $4428 \mathrm{~kg} / \mathrm{m}^{3}$
For the fixation cement we assigned the following parameters: Young's modulus $\mathrm{E}=2150 \mathrm{~N} / \mathrm{mm}^{2}$, Poisson's ratio $=0.48$, density $=1100 \mathrm{~kg} / \mathrm{m}^{3}$.

We discretized the entire virtual model into nodes and elements, we applied a $2100 \mathrm{~N}$ load force (3 times the average weight of a patient) on the surfaces of the polyethylene insert and a gravitational acceleration of $9.81 \mathrm{~m} / \mathrm{s}^{2}$ along the longitudinal axis of the of the tibial bone.

We analyzed the mechanical behavior of both assemblies (healthy tibia-knee implant, respectively impaired tibia-knee implant) under static stress, linear and nonlinear dynamic stress and fatigue testing conditions.

\section{Results of the Static Stress Analysis for the Healthy Tibial Bone-Knee Implant Assembly}

The highest stress (von Mises) values obtained under static load conditions are 21.9 MPa and were measured on the polyethylene insert, which indicates that this component, like the lateral meniscus, absorbs part of the stresses, as can be seen in Fig. 3.

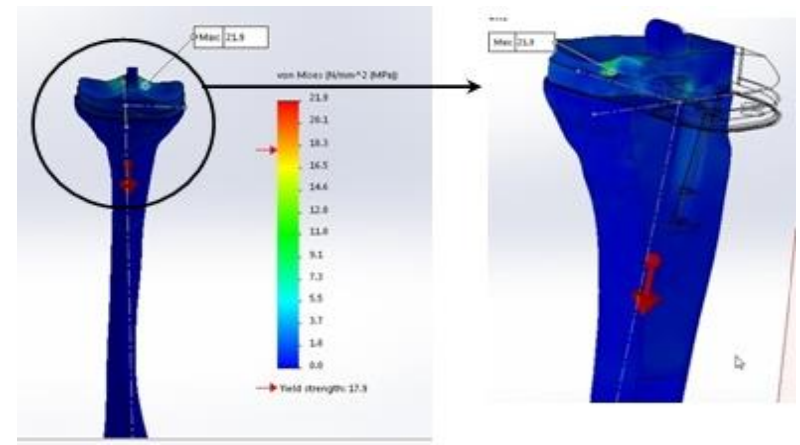

Figure 3: Von Mises stress values (MPa). Detail section through the assembly, with the corresponding maximum stress values

The largest displacement values measured 0.117 $\mathrm{mm}$ and were found on the polyethylene insert.

These displacements propagate progressively through the tibial metal component (Fig. 4). On the $\mathrm{Z}$-axis, the largest displacements are up to $0.098 \mathrm{~mm}$.

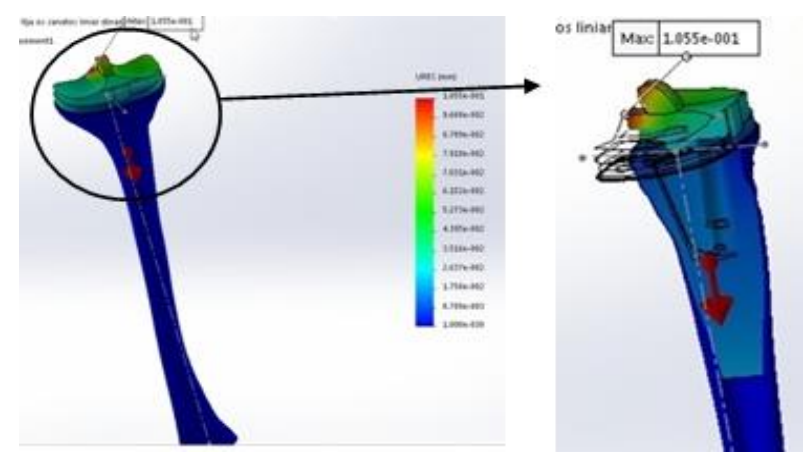

Figure 4: URES displacements ( $\mathrm{mm}$ ). Sectional detail 
As for the contact pressure between the tibialcomponent, the cement and the proximal part of the tibial bone (near the implant), a high pressure was measured between these components, which indicates a high pressure resistance of $13.1 \mathrm{MPa}$, Fig. 5.

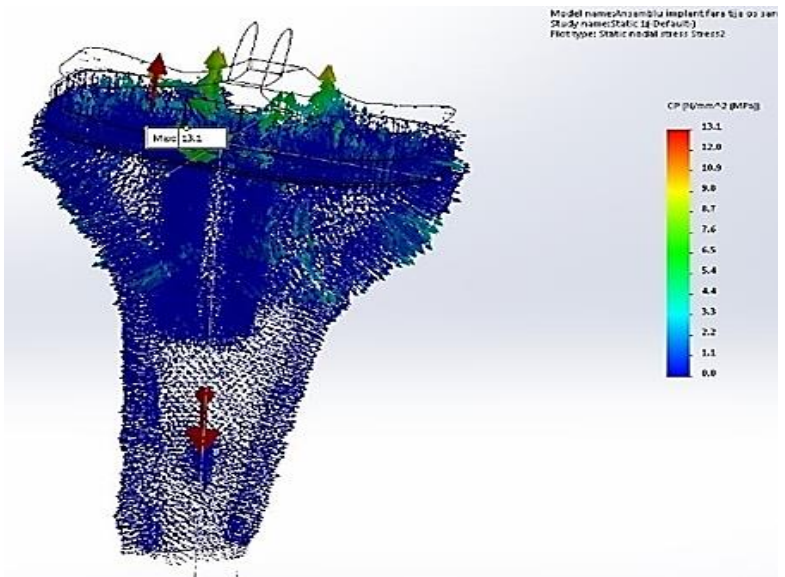

Figure 5: Contact pressure results $\mathrm{MPa}$

\section{Results of The Fatigue Resistance Analysis for the Healthy Tibial Bone- Knee Implant Assembly}

After assigning the initial conditions, we used the software to add an "event" which enabled us to analyze the resistance of the implanted bone when going through 700,000 functioning cycles.

After the event simulation, we obtained the following results:

- Damage percentage - the percent of the bone assembly that is damaged after going through the selected number of cycles, seen as a whole. In our case, the maximum damage percentage was $41.7 \%$ and was measured on the polyethylene insert, Fig. 6a.

- Total Life (cycle) - the total number of cycles the assembly withstands. In the case under study, the maximum number of cycles that the tibial boneknee implant assembly can withstand is 1.000.000. Therefore, the assembly is able to withstand the 700,000 cycles it has been subjected to. Fatigue occurs in the back area of the polyethylene insert after 83,500 cycles, Fig. 6-b.

- Load Factor - the load factor under the impact of the maximum number of cycles the assembly is subjected to, and the force applied to it. It is noted that the red area is found at the bottom of the tibia, which is confirmed by the fact that in most cases the tibial bone breaks above the ankle - Fig. 6c.

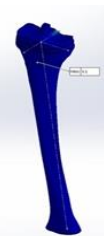

a)

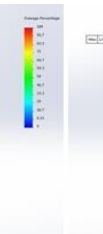

(2)

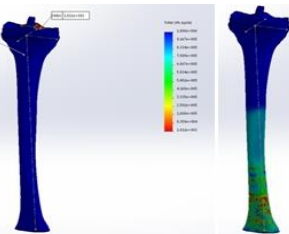

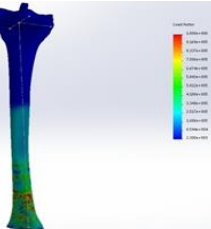

Figure 6: Fatigue analysis results a) Damage percentage b) Total life (maximum number of cycles) c) Load factor

\section{Results of the Linear Dynamic Stress Analysis for the Healthy Tibial Bone- Knee Implant Assembly}

For the purpose of this analysis, we will apply a $214 \mathrm{kgf}(2100 \mathrm{~N})$ inertial mass distributed along the longitudinal axis of the tibia. This force will be applied to the assembly for one second, Fig. 7.

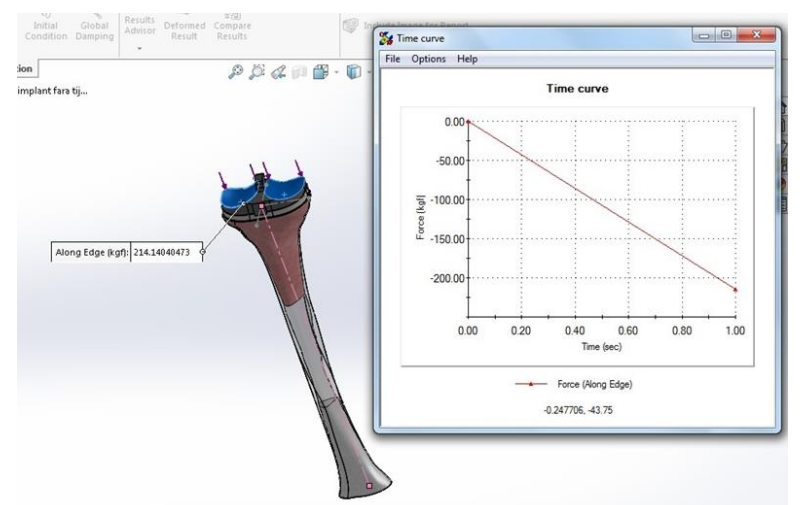

Figure 7: Applying the force. Graph showing the force applied to the assembly for a period of one second

The highest von Mises stress values measure 43.2 MPa and are found at the contact area between the metallic tibial component and the proximal part of the tibial bone, Fig. 8 .

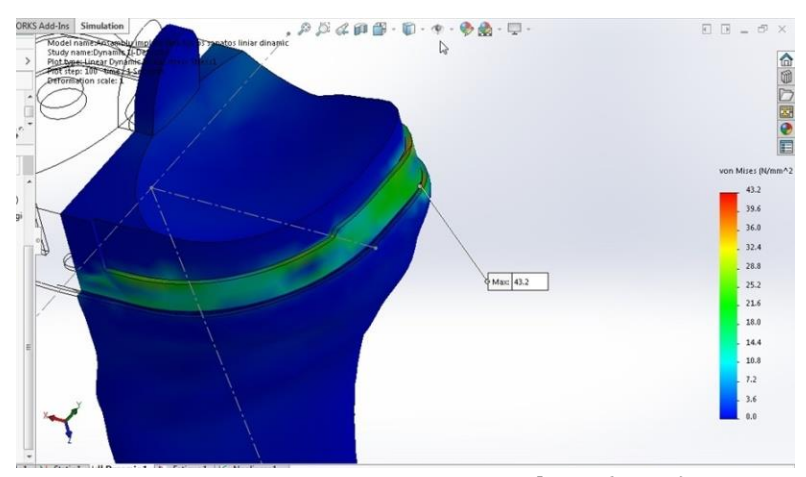

Figure 8: Von Mises stress values (MPa)

The largest displacements are found on the Z-axis, on the polyethylene insert, and measure $0.165 \mathrm{~mm}$. 


\section{Results of the Nonlinear Dynamic Stress Analysis for the Healthy Tibial Bone-Knee Implant Assembly}

The force applied is $2100 \mathrm{~N}$ on the surfaces of the polyethylene insert, along the longitudinal axis, Fig. 9-a. The curve showing the force variation for a period of one second is nonlinear, as can be seen in Fig. 9-b.

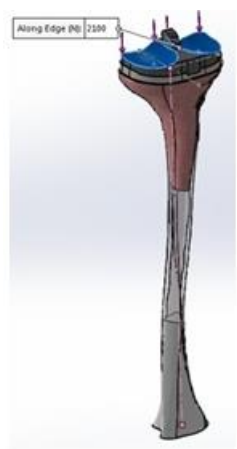

a)

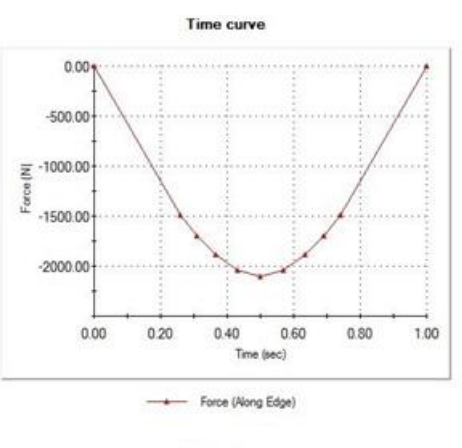

b)
Figure 9: Applying the load force: a) force directionalong the longitudinal axis b) graph showing the force variation with time

The highest stress values are found on the edge of the tibial component, at the contact with the polyethylene insert, and measure 84.4 MPa, Fig. 10.

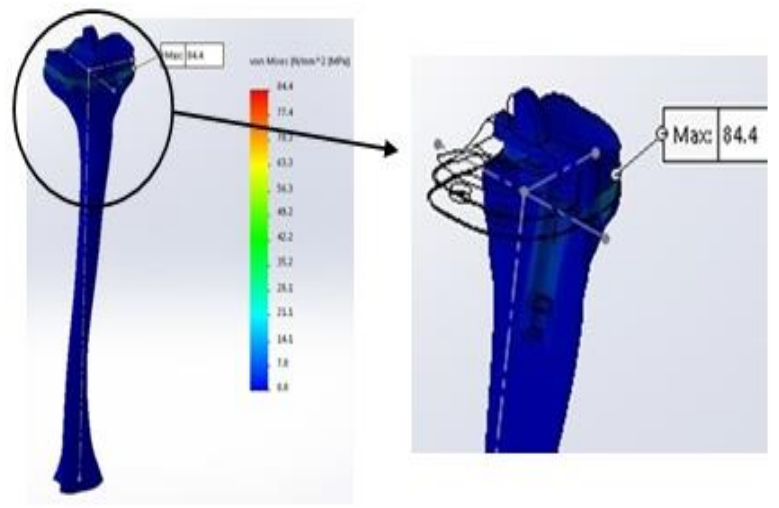

Figure 10: Von Mises stress values (MPa), von Mises stress (MPa) detail

In this case also, the displacements are found on the polyethylene insert and measure up to $0.151 \mathrm{~mm}$. The displacement values decrease gradually towards the tibial component, Fig. 11.

The detail below shows a section through the assembly and the displacements measured on the polyethylene insert.

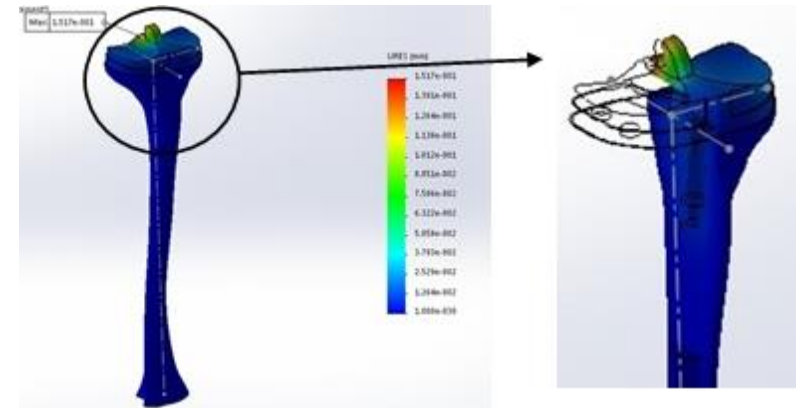

Figure 11: URES Displacement ( $\mathrm{mm}$ ) Result, sectional detail of the assembly

The equivalent displacements are very small, up to $5.93 \times 10-6 \mathrm{~mm}$.

The next step was to analyze the osteoporotic bone under the same stress conditions, in order to determine its behavior in comparison with that of the healthy bone.

For this purpose, we altered the mechanical properties of the healthy spongy bone the implant is fitted into and we assigned the following material properties to the osteoporotic spongy bone:

Young's modulus E $=1000 \mathrm{~N} / \mathrm{mm}^{2}$, Poisson's ratio $v=0.2$, density $\rho=400 \mathrm{~kg} / \mathrm{m}^{3}$, Tensile strength $1.1 \mathrm{~N} / \mathrm{mm}^{2}$, Compressive strength $1.7 \mathrm{~N} /$ $\mathrm{mm}^{2}$.

\section{Results of the Static Stress Analysis for the Impaired Tibial Bone-Knee Implant Assembly}

The highest stress values were found on the polyethylene insert and measure 21.3 MPa (Fig. 12).

As can be noticed from the detail showing a section through the bone, these stresses propagate also along the tibial component.

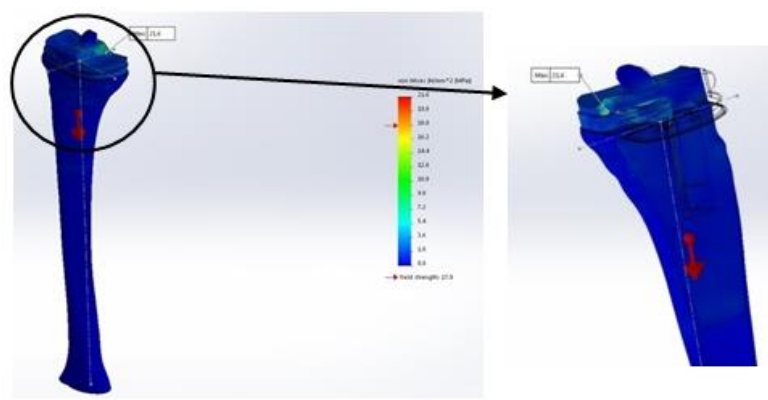

Figure 12: Von Mises stress values (MPa), sectional detail of the bone

The pressure values found at the contact between the tibial component, the cement and the proximal part of the tibial bone are low (12.8 MPa, Fig. 13). 


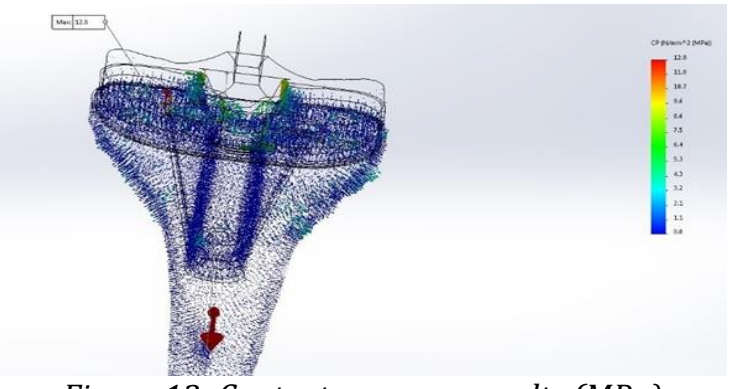

Figure 13: Contact pressure results (MPa)

The highest displacement values are found on the polyethylene insert, they measure $0.117 \mathrm{~mm}$ and are transferred progressively to the other components. The displacements on the Z-axis are lower than the overall values and measure $0.97 \mathrm{~mm}$.

\section{Results of the Fatigue Resistance Analysis for the Impaired Tibial Bone- Knee Implant Assembly}

After assigning the initial conditions, we used the software to add an "event" which enabled us to analyze the resistance of the impaired bone when going through 700,000 cycles. We configured the Damage percentage parameter for the entire model and we found that our assembly is damaged to a proportion of minimum $70 \%$ and maximum $91.7 \%$ in the area of the polyethylene insert, Fig. 14-a.

We also found that the maximum number of cycles the impaired bone-knee implant assembly can withstand is $1,000,000$ cycles.

Fatigue occurs in the back area of the polyethylene insert after 60,441 cycles, Fig. 14-b.

The load factor reaches its maximum values at the bottom of the tibial bone, which confirms that in most of the cases the tibial bone breaks above the ankle, Fig. 14-c.

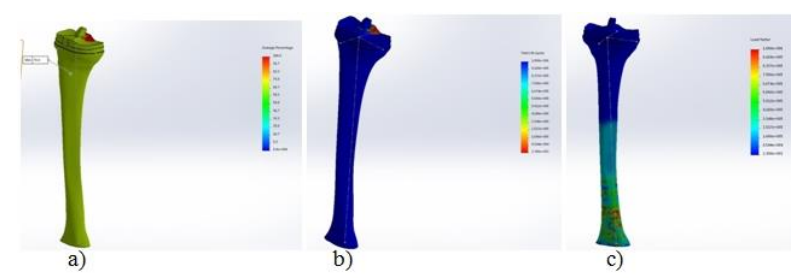

Figure 14: Fatigue analysis results. a) damage percentage b) maximum number of cycles (total life) c) load factor

\section{Results of the Linear Dynamic Stress Analysis for the Impaired Tibial Bone- Knee Implant Assembly}

The inertial mass applied is $214 \mathrm{kgf}(2100 \mathrm{~N})$ distributed along the longitudinal axis of the tibia, increasing linearly, for a period of one second, Fig. 15.
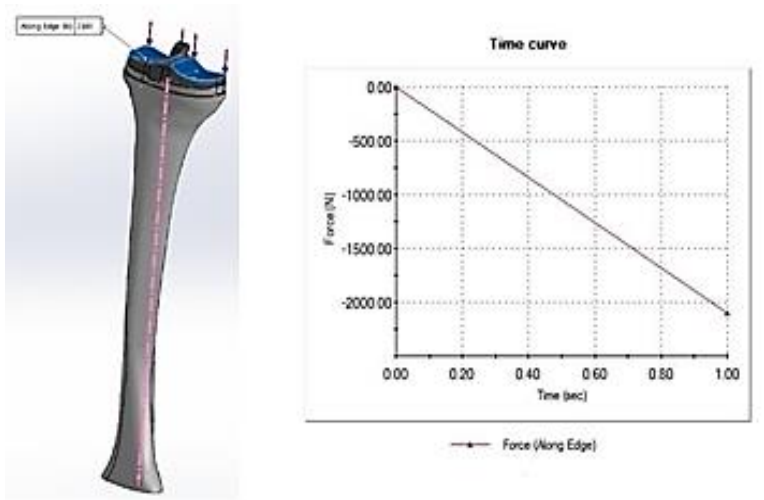

Figure 15: Left: applying the force; Right: time-force graph for a force applied for one second

The highest von Mises stress values, measuring 28.4 $\mathrm{MPa}$, are found on the edge of the tibial component, as seen in Fig. 16-a, and on the polyethylene insert as well.

In this case also, the largest displacement values are found on the polyethylene insert and measure $0.154 \mathrm{~mm}$, as seen in Fig. 16-b. These displacements are transferred to the tibial component as well. On the Z-axis the displacements measure maximum $0.150 \mathrm{~mm}$, as seen in Fig. 16-c. The displacements are found on the polyethylene insert and at the contact with the tibial component.

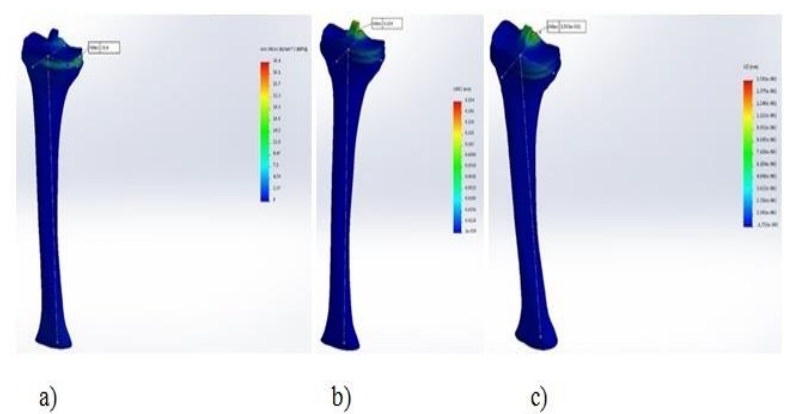

Figure 16: a) Von Misses stress values (MPa), b) URES displacements ( $\mathrm{mm}), \mathrm{c}$ ) URES displacements on the Zaxis ( $\mathrm{mm})$

\section{Results of the Nonlinear Dynamic Stress Analysis for the Impaired Tibial Bone-Knee Implant Assembly}

The $2100 \mathrm{~N}$ force is applied along the longitudinal axis of the tibial bone, on the selected surfaces of the polyethylene insert.

The force varies in a nonlinear manner, and the graph representing the force applied for a period of one second is shown in Fig. 17. 

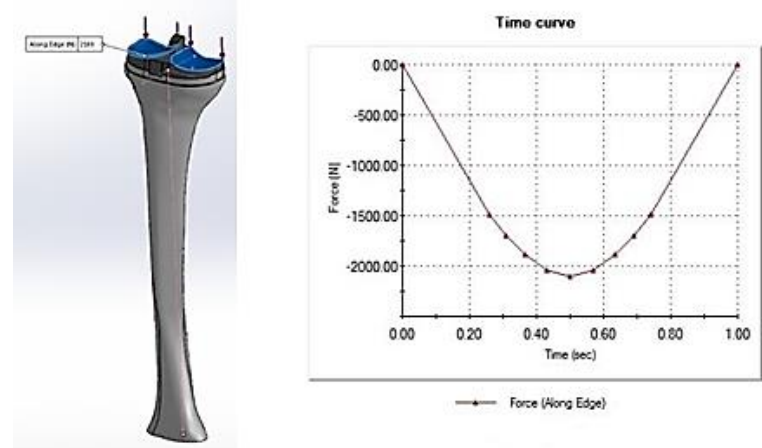

Figure 17: Applying the force. The time-force graph showing the force variation over one second

The highest stress values, measuring $32 \mathrm{MPa}$, are found on the edges of the tibial component and of the cortical bone, but are also transferred through the polyethylene insert, as seen in Fig. 18.

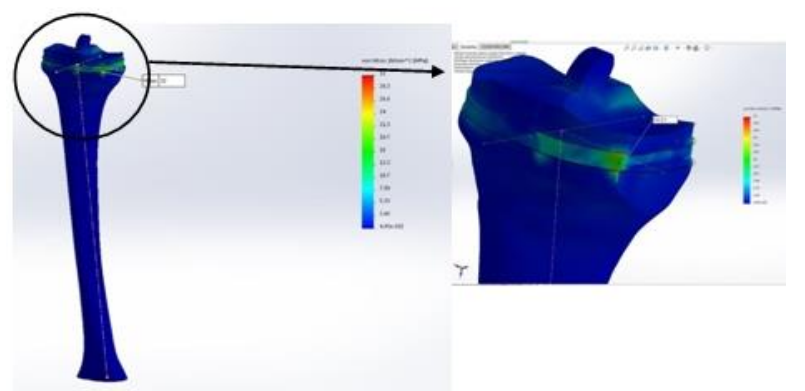

Figure 18: Von Misses stress values (MPa), detail of von Misses stress results (MPa)

In this case also, the displacements are located on the polyethylene insert and they measure $0.068 \mathrm{~mm}$. The displacement values decrease gradually towards the tibial component.

The maximum equivalent displacement values measure $1.139 \times 10^{-5} \mathrm{~mm}$.

\section{Conclusions. Future Research Directions}

The results obtained from the analysis performed on a healthy bone and a diseased bone subjected to static stress, fatigue, and linear and nonlinear dynamic stress, confirm that the healthy bone has a higher resistance than the diseased one, under the same stress conditions. These results allow us to substantiate, based on the bone density values, the predictions as to the long-term behavior of an implanted bone. Predictive biomechanical studies based on the finite element method may provide surgeons with valuable information that can help them choose the most appropriate type of implant. As a future research direction, it will be necessary to perform the finite element analysis of an impaired bone-knee implant assembly with a tibial augmentation, to compare the results of such analysis to the those obtained from the analysis performed on the impaired bone-knee implant assembly without a tibial extension (from the current paper) and to determine which option is best suited to be used from the very beginning (at the moment of the primary surgery): the implant that includes the extension or the implant without the extension.

If further to the comparative analysis it turns out that using the implant without an extension will result, after a certain number of functioning cycles, in a high degree of damage, which exceeds the damage that occurs when using an implant with extension, then the conclusion would be that it is advisable to choose the implant with the tibial extension at the moment of the primary surgical procedure.

Otherwise, the revision surgery required to add the tibial extension will come soon after the primary surgery.

\section{References}

[1] Cristea Şt., Prundeanu A., Groseanu Fl. and Gârtone D., The Role of Arthroscopy in MiniInvasive Treatment of Tibial Plateau Fractures, Chapter from the book Modern Arthroscopy, pp. 225-236, (2011).

[2] Cofaru I.I., Summary of the PhD Thesis, Researches regarding the biomechanics of the axial deviations of the human lower member and the development of the correspondent surgical devices, (2013).

[3] Nicolescu C.M., Bumbac M., Mihai S., Gheboianu A.I., Balasa M.C., Filip V., Cuculici S., Cristea S., Pantu C., X-RAY diffraction and nanoindentation characterization of bone tissue affected by severe osteoarthritis, Journal of Science and Arts, ISSN: 1844 - 9581, Year 18, No. 1(42), pp. 265-274, (2018).

[4] LNCS Homepage, http://mihairascu.ro/protezatotala-a-genunchiului/, last accessed 2019/05/29

[5] Balaşa M.C., Mihai S., Filip V., Negrea A.D., Tomescu G., Modelling the tibial bone using CAD techniques, starting from the 3D scan model, International Journal of Mechatronics and Applied Mechanics, Issue 3, pp. 217-223, (2018).

[6] Balaşa M.C., Cuculici Ş., Panţu C., Mihai S., Negrea A.D., Zdrafcu M.O., Leţ D.D., Filip V., Cristea Ş., Using 3D scanning techniques in orthopedic systems modeling, The Scientific Bulletin of VALAHIA University MATERIALS and MECHANICS -Vol. 15, No. 13, pp. 41-47, (2017). 